\title{
Cardioprotective Solutions Exposure For 1 Hour in Hypoxia and Low Temperatures Affects Vascular Reactivity Differently
}

Priscila Rossi de Batista², PhD; Dalton Valentim Vassallo ${ }^{1,2}, \mathrm{MD}$, PhD; Maylla Ronacher Simões , PhD; Melchior Luiz

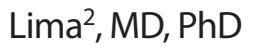

DOI: $10.21470 / 1678-9741-2020-0320$

\begin{abstract}
Introduction: Heart preservation benefits cardiac performance after operations decreasing morbidity but the contribution of the vascular reactivity has been neglected.

Objective: We evaluated whether cardioprotective solutions, Krebs-Henseleit (KH), Bretschneider-HTK (BHTK), St. Thomas No. 1 (STH-1), and Celsior (CEL), affect vascular reactivity.

Methods: Aortic rings from Wistar rats were used in two protocols. First, the rings were exposed to BHTK, STH-1 or CEL for 1 hour of hypoxia at $37{ }^{\circ} \mathrm{C}$. Second, the rings were exposed to $10^{\circ} \mathrm{C}$ or $20^{\circ} \mathrm{C}$ for 1 hour under hypoxia. After treatment, the rings were immersed in $\mathrm{KH}$ at $37{ }^{\circ} \mathrm{C}$, endothelial integrity was tested and concentration-response curves to phenylephrine were performed.

Results: In the first protocol, the solutions did not damage the endothelium; $\mathrm{CEL}$ and $\mathrm{BHTK}$ reduced $\mathrm{KCl}$-induced contractions but not STH-1; only CEL and BHTK reduced vascular reactivity; there was a positive correlation between $\mathbf{R}_{\max }$ and $\mathrm{KCl}$ concentration.
\end{abstract}

At $20^{\circ} \mathrm{C}$, 1 hour under hypoxia, the solutions produced similar $\mathrm{KCl}$-induced contractions without endothelial damage. CEL, BHTK and STH-1 decreased vascular reactivity. At $10{ }^{\circ} \mathrm{C}$, STH-1 increased reactivity but CEL and BHTK decreased. After 1 hour under hypoxia in CEL or BHTK solutions, reactivity was similar at different temperatures. At $20^{\circ} \mathrm{C}$, endothelial damage after exposure to STH-1 produced more vasoconstriction than CEL and BHTK. However, at $10^{\circ} \mathrm{C}$, endothelial damage after CEL and BHTK exposure elicited more vasoconstriction while STH-1 showed a small vasoconstrictor response, suggesting endothelial damage.

Conclusion: STH-1 decreased reactivity at $20^{\circ} \mathrm{C}$ and increased at $10^{\circ} \mathrm{C}$. CEL promoted greater endothelial modulation at $10{ }^{\circ} \mathrm{C}$ than at $20{ }^{\circ} \mathrm{C}$, while STH-1 promoted higher modulation at 20 ${ }^{\circ} \mathrm{C}$ than at $10{ }^{\circ} \mathrm{C}$. Vascular tone was reduced by CEL and BHTK exposure, also depending on the $\mathrm{KCl}$ concentration.

Keywords: Vasoconstriction. Vasoconstrictor Agents. Hypoxia. Endothelium. Temperature. Phenylephrine.

\begin{tabular}{|c|c|c|c|}
\hline \multicolumn{4}{|c|}{ Abbreviations, acronyms \& symbols } \\
\hline $\mathrm{ACh}$ & $=$ Acetylcholine chloride & KH & $=$ Krebs-Henseleit \\
\hline ATP & $=$ Adenosine triphosphate & $\mathrm{KH}_{2} \mathrm{PO}_{4}$ & = Monopotassium phosphate \\
\hline AUC & $=$ Area under the curve & $\mathrm{mM}$ & $=$ Millimolar \\
\hline ANOVA & $=$ Analysis of variance & $\mathrm{MgSO}_{4}$ & $=$ Magnesium sulfate \\
\hline BHTK & $=$ Bretschneider-HTK & $\mathrm{NaCl}$ & $=$ Sodium chloride \\
\hline $\mathrm{CaCl}_{2}$ & $=$ Calcium chloride & $\mathrm{NaHCO}_{3}$ & = Sodium bicarbonate \\
\hline CEL & $=$ Celsior & PHE & = Phenylephrine \\
\hline $\mathrm{CO}_{2}$ & $=$ Carbon dioxide & $\mathbf{R}_{\max }$ & $=$ Maximal response \\
\hline dAUC & $=$ Differences in areas under the curves & SEM & $=$ Standard error of the mean \\
\hline EDTA & $=$ Ethylenediaminetetraacetic acid & STH-1 & $=$ St. Thomas No. 1 \\
\hline HTK & = Histidine-tryptophan-ketoglutarate & $\mu \mathrm{M}$ & $=$ Micrometer \\
\hline $\mathrm{KCl}$ & $=$ Potassium chloride & & \\
\hline
\end{tabular}

'Department of Physiological Sciences, Federal University of Espírito Santo, Vitória, ES, Brazil.

${ }^{2}$ Centro de Ciências da Saúde de Vitória-EMESCAM, Vitória, ES, Brazil.

This study was carried out at the Department of Physiological Sciences, Federal University of Espírito Santo, Vitória, ES, Brazil.
Correspondence Address:

Maylla Ronacher Simões

iD https://orcid.org/0000-0003-1395-3938

Department of Physiological Sciences, Federal University of Espírito Santo Av. Marechal Campos, 1468, Vitória, ES, Brasil - Zip code: 29040-095

E-mail:yllars@hotmail.com 


\section{INTRODUCTION}

Myocardial protection has great relevance for the advances in heart transplantation ${ }^{[1]}$. Cardioprotection refers to strategies used to attenuate or prevent myocardial dysfunction during or after cardiac surgery. To achieve this goal, cardioprotective and cardioplegic solutions have been used. The mechanism of a cardioprotective solution is based upon three principles: a) Hypothermic stop of the metabolism; b) A physical and biochemical environment to maintain viable tissue structure during the hypothermic stop of the metabolism; and c) Minimizing the effects of the reperfusion injury ${ }^{[2]}$.

The main purpose of the composition of preservation solutions is to induce a fast depolarization of the cardiac myocyte membrane by reducing the transmembrane $\mathrm{K}^{+}$gradient ${ }^{[3]}$. This procedure stops the mechanical and electrical activity of the heart. Saline solutions with composition similar to the extracellular fluid such as Ringer's lactate and Hartmann were initially used for myocardial protection but were considered inadequate to maintain the myocardial protection and its function ${ }^{[4]}$.

To improve contractile function after periods of ischemia, hypothermic procedures have been used ${ }^{[5-7]}$. Hypothermia reduces energy consumption by protecting cellular metabolism and improving resistance to ischemia during cardioplegic cardiac arrest ${ }^{[8]}$. The increase in the supply and energy demand ratio during ischemia is generally attributed to hypothermic protection and hypothermia also provides an important reduction of oxidative stress induced by ischemia and reperfusion ${ }^{[9]}$.

So far, cardiac protection for transplantation has been focused mainly on the cardiac mechanical activity but studies about the effects of solutions used on vascular reactivity have been neglected. Some focus was given to the coronary vessels ${ }^{[10,11]}$ but not to the systemic vasculature and under different temperatures. If these protective solutions damage the vessels, they can be harmful to the heart blunting its mechanical recovery and increasing afterload during ventricular ejection. Therefore, we aimed to study how preservation solutions used in cardiac transplantation affect vascular reactivity. Our attempt was to investigate whether the Krebs-Henseleit $(\mathrm{KH})$, Bretschneider-HTK (BHTK), St. Thomas No. 1 (STH-1) and Celsior (CEL) solutions might affect the vascular reactivity of aortic rings, a conductance vessel, submitted to 1 hour of hypoxia, and if 1 hour of hypoxia at 10 and $20^{\circ} \mathrm{C}$ alters vascular reactivity, producing or avoiding endothelial damage. Results will enable us to select which solutions induce non-deleterious changes for further investigation.

\section{METHODS}

\section{Animals and Treatment}

Male Wistar rats (260-300 g, N=64) were used for these studies. The care and use of laboratory animals were in accordance with the National Institutes of Health guidelines, and all experiments were conducted in accordance with the guidelines for biomedical research as stated by the Brazilian Societies of Experimental Biology and were approved by the
Institutional Ethics Committee of the Health Sciences Center of Vitória (CEUA-EMESCAM 004/2007). All rats had free access to water and were fed with rat chow ad libitum. The rats were anesthetized with pentobarbital (35 mg/kg, i.p.) and euthanized by exsanguination. The thoracic aortas were carefully dissected, placed in aerated $\mathrm{KH}$ solution and fat and connective tissue were removed. For vascular reactivity experiments, the aortas were divided into cylindrical segments $4 \mathrm{~mm}$ long.

Two protocols were performed. In the first protocol, the rings were immersed in $\mathrm{KH}, \mathrm{BHTK}, \mathrm{STH}-1$ and $\mathrm{CEL}$ solutions for 1 hour of hypoxia, at $37^{\circ} \mathrm{C}$. In the second protocol, the rings were immersed in BHTK, STH-1 and CEL solutions, at 10 or $20^{\circ} \mathrm{C}$ for 1 hour of hypoxia, except for the $\mathrm{KH}$ solution, in which the rings were kept at $37^{\circ} \mathrm{C}$, and gassed with $95 \% \mathrm{O}_{2}-5 \% \mathrm{CO}_{2} \mathrm{pH} 7.4$.

\section{Vascular Reactivity Measurements Hypoxia Protocol}

This protocol was performed first to define if the selected cardioprotective solutions could cause any vascular harm. After anaesthesia, the descending thoracic aorta was removed and placed in a Petri dish immersed in $\mathrm{KH}$ solution. The rings were immediately placed in the solutions of interest (CEL, BHTK, or STH-1) without aeration with a carbogenic mixture for 1 hour at $37^{\circ} \mathrm{C}$. After 1 hour of hypoxia, the aortic segments were mounted between two parallel wires in organ baths containing $\mathrm{KH}$ solution (in mM: $124 \mathrm{NaCl}, 4.6 \mathrm{KCl}, 2.5 \mathrm{CaCl}_{2}, 1.2 \mathrm{MgSO}_{4}, 1.2$ $\mathrm{KH}_{2} \mathrm{PO}_{4}, 0.01 \mathrm{EDTA}, 23 \mathrm{NaHCO}_{3}$ ) at $37^{\circ} \mathrm{C}$ and gassed with $95 \%$ $\mathrm{O}_{2}-5 \% \mathrm{CO}_{2}$ ( $\mathrm{pH}$ 7.4). The arterial segments were stretched to an optimal resting tension of $1 \mathrm{~g}$. The tension was recorded using a force transducer (TSD125C, CA, USA) connected to an acquisition system (MP100A, BIOPAC System, Inc., Santa Barbara, USA). After a 45 minutes equilibration period, all aortic rings were initially exposed twice to $75 \mathrm{mM} \mathrm{KCl}$. The first exposure checks their functional integrity, and the second exposure assesses the maximal tension. Next, endothelial integrity was tested with acetylcholine (ACh, $10 \mu \mathrm{M})$ in segments previously contracted with phenylephrine $(1 \mu \mathrm{M})$. A relaxation $\geq 90 \%$ was considered indicative of the functional integrity of the endothelium. After a washout period (30 min), concentration-response curve for phenylephrine $(0.1 \mathrm{nM}-30 \mathrm{mM})$ was performed and the tension was measured once a plateau was attained.

\section{Temperature Protocols}

After anaesthesia, the descending thoracic aorta was removed and placed in a Petri dish immersed in $\mathrm{KH}$ solution. Then, the rings were immediately placed in the solutions of interest (CEL, BHTK, or STH-1), without carbogenic mixture, for 1 hour at a temperature of 20 or $10^{\circ} \mathrm{C}$; at the end of this period, the rings were placed in the system under the normal experimental condition, $\mathrm{KH}$ solution, with carbogen, at a constant temperature of $37^{\circ} \mathrm{C}$ (control condition).

Control Group: After cutting, the rings were placed in the control condition described above during the same period (1 h). Thus, all groups were submitted to the same experimental protocols described above: a) Viability test of vascular smooth 
muscle and endothelium; b) Evaluation of vascular reactivity from concentration-response curves to phenylephrine ( $\mathrm{PHE}$, 10-10 to 10-4 M); c) This same evaluation of vascular reactivity was performed in the absence of the endothelium (E), after mechanical injury of the rings before being placed in the system, to comparatively study the endothelium participation.

\section{Drugs and Reagents}

L-phenylephrine hydrochloride, ACh and sodium pentobarbital were purchased from Sigma-Aldrich (St. Louis, USA). Salts and reagents used were of analytical grade from Sigma-Aldrich and Merck (Darmstadt, Germany).

\section{Statistical Analyses}

Results are expressed as the mean \pm SEM of the number of rats studied; differences were analysed using Student's t-test or one or two-way ANOVA followed by a Bonferroni test. A $P<0.05$ was considered significant. Contractile responses to phenylephrine were expressed in absolute values. For each concentration-response curve, the maximal response $\left(R_{\max }\right)$ and the concentration of agonist that produced $50 \%$ of the maximal response (log $\left.\mathrm{EC}_{50}\right)$ were calculated using non-linear regression analysis (GraphPad Prism, GraphPad Software, Inc., San Diego, $(A)$. The sensitivities of the agonists were expressed as $\mathrm{pD}_{2}$ $\left(-\log \mathrm{EC}_{50}\right)$. To compare the effects of different solutions and temperatures, some results were expressed as differences in the areas under the concentration-response curves (dAUC) in both the control and experimental groups. AUCs were calculated from individual concentration-response curve plots: the differences are expressed as the percentage of the control AUC.

\section{RESULTS}

Our first attempt was to investigate whether 1 hour of hypoxia at $37^{\circ} \mathrm{C}$ would affect the vascular contraction induced by $75 \mathrm{mM}$ of $\mathrm{KCl}$. Figure 1 shows that, after hypoxia, $\mathrm{KCl}-$ induced contractions were different among the solutions used. Krebs and STH-1 did not change $\mathrm{KCl}$-induced contraction, but CEL and BHTK solutions reduced the contractions of aortic rings.

However, at 20 and $10{ }^{\circ} \mathrm{C}$, all groups exposed to STH-1, CEL and BHTK solutions presented, after 1 hour of hypoxia, similar $\mathrm{KCl}$-induced contractions (in g: CEL at $20^{\circ} \mathrm{C}$ $3.29 \pm 0.2$, at $10{ }^{\circ} \mathrm{C} 3.16 \pm 0.2$; STH- 1 at $20^{\circ} \mathrm{C} 32 \pm 0.2$, at $10^{\circ} \mathrm{C} 3.77 \pm 0.1$; BHTK at $20^{\circ} \mathrm{C} 3.36 \pm 0.2$, at $\left.10^{\circ} \mathrm{C} 3.38 \pm 0.2\right)$. In addition, hypoxia for 1 hour, in both protocols, did not affect the functional endothelial integrity. The relaxation induced by ACh $(10 \mu \mathrm{M})$ was preserved in segments exposed to different solutions, previously contracted with phenylephrine $(1 \mu \mathrm{M})$ (results not shown).

Hypoxia at $37^{\circ} \mathrm{C}$ did not change the capacity of aortic rings, previously exposed to preservation solutions and then incubated with Krebs, to contract in response to phenylephrine (hypoxia at $37^{\circ} \mathrm{C}$, Figure 2). However, concentration-response curves to phenylephrine showed a reduction in $\mathrm{R}_{\max }$ after 1 hour of hypoxia under CEL and BHTK solutions. Concerning STH-1 solution, aortic rings showed vascular reactivity to phenylephrine similar to Krebs control (hypoxia at $37^{\circ} \mathrm{C}$, Figures $2 \mathrm{~A}, \mathrm{~B}$ and $\mathrm{C}$ ).

As mentioned above, $\mathrm{CEL}$ and $\mathrm{BHTK}$ solutions reduced the vascular reactivity of aortic rings to phenylephrine. To understand these results, we also investigated a putative relationship between $R_{\max }$ and the composition of the solutions. There was no correlation between calcium concentratio $n$ and $R_{\max }$ (results not shown). However, there was a positive correlation between $R_{\max }$ and the potassium concentration of the solutions (hypoxia at $37^{\circ} \mathrm{C}$, Figure 3), suggesting that the maintenance of a reduced vascular tone depends on the smaller amount of potassium in the solutions.

Figure 4 shows the concentration-response curves to phenylephrine and the effects of exposing aortic rings to CEL solution at 10 and $20^{\circ} \mathrm{C}$ compared to control Krebs solution at $37^{\circ} \mathrm{C}$. Sensitivity was reduced at both temperatures (Figures $4 \mathrm{~A}$ and $\mathrm{B})$ but without differences when compared between them (Figure 4C). However, concentration-response curves to phenylephrine, at both temperatures, did not show changes of $\mathrm{R}_{\text {max }}$. Regarding the BHTK solution, a similar behaviour as CEL was observed (Figures 4D, E and F). These findings suggest that, if exposed to vasoconstrictors, these vessels would preserve a better flow by developing smaller contractions.

Regarding the $10{ }^{\circ} \mathrm{C}$ and $20{ }^{\circ} \mathrm{C}$ protocol, STH-1 solution showed an increased sensitivity of the vascular reactivity to phenylephrine at $20^{\circ} \mathrm{C}$ when compared to control Krebs solution at $37^{\circ} \mathrm{C}$, but not at $10^{\circ} \mathrm{C}\left(10\right.$ and $20^{\circ} \mathrm{C}$ protocol, Figures $5 \mathrm{~A}$ and

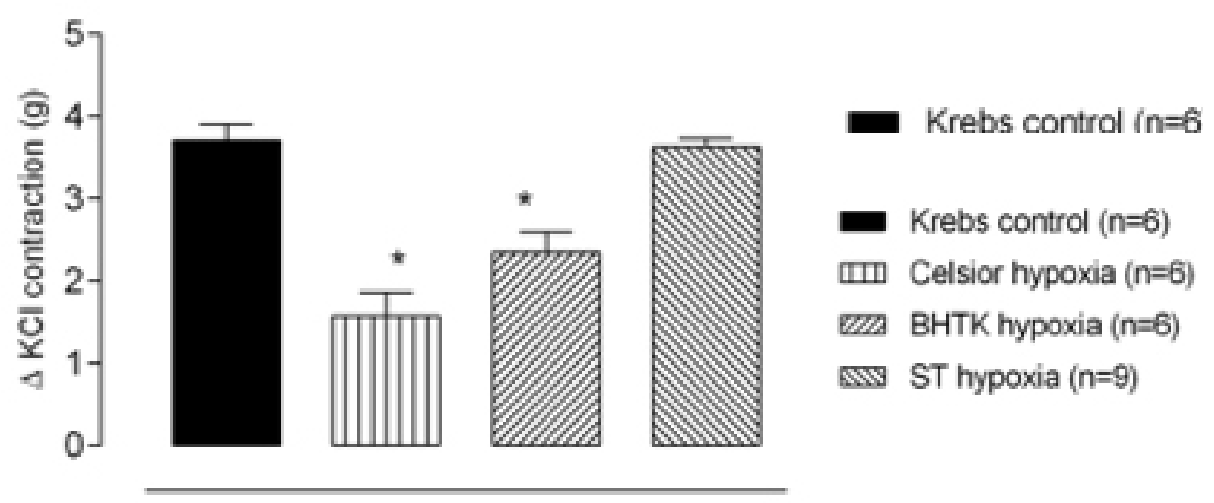

Fig. 1 - Contractile response to potassium chloride ( $\mathrm{KCl}-75 \mathrm{mM})$ in isolated aortic rings after 1 hour of hypoxia at $37^{\circ} \mathrm{C}$ in Krebs-Henseleit (Krebs control), Celsior, Bretschneider-HTK (BHTK) or St. Thomas (ST) solutions. Results expressed as mean \pm SEM. ${ }^{*} P<0,05$ vs. Krebs-Henseleit (control), oneway ANOVA, Turkey test. The numbers in parentheses indicate the number of specimens used. 

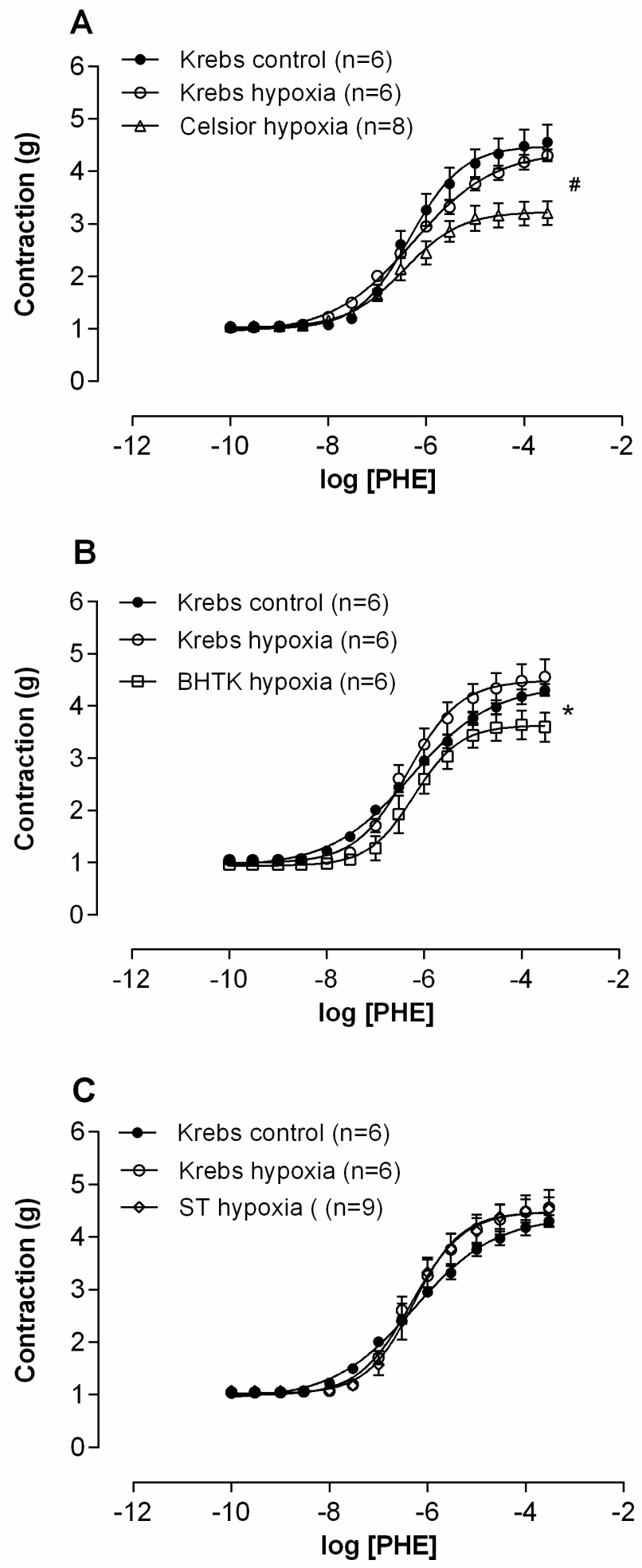

Fig. 2 - Concentration-response curves to phenylephrine (PHE) in isolated aortic rings of Wistar rats after incubation for 1 hour in hypoxia with Celsior (A), Bretschneider-HTK (BHTK) (B) and St. Thomas (ST) (C) solutions, compared to the Krebs control solution, at $37^{\circ} \mathrm{C}$ and after 1 hour of hypoxia. Results expressed as mean \pm SEM. ${ }^{*} P<0.05$ for comparison of Rmax vs. control Krebs, t-test. The numbers in parentheses indicate the number of specimens used.

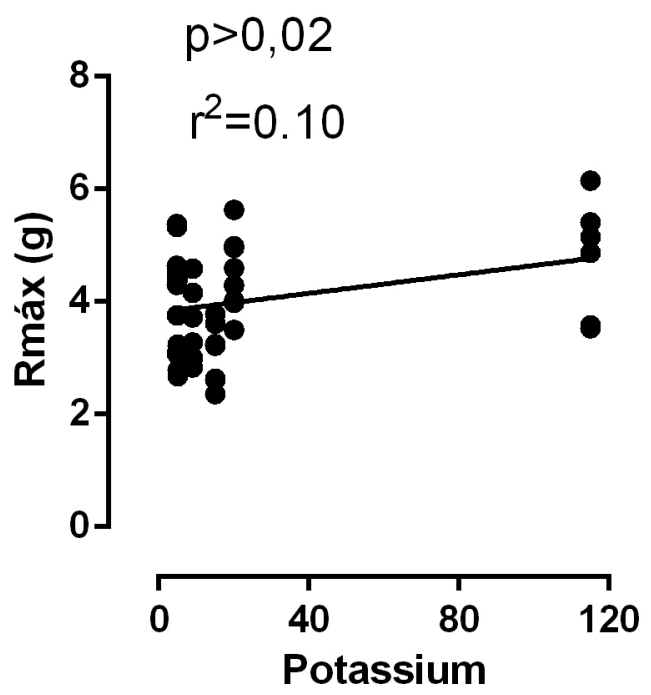

Fig. 3 - Correlation between Rmax and the potassium concentration of the studied solutions Krebs-Henseleit (Krebs control), Celsior, Bretschneider-HTK and St. Thomas-1.P<0.02.

B). Moreover, $\mathrm{R}_{\max }$ increased after exposure to $\mathrm{STH}-1$ solution at $10{ }^{\circ} \mathrm{C}$ but not at $20^{\circ} \mathrm{C}$. However, comparing concentrationresponse curves to phenylephrine at both temperatures, $\mathrm{R}_{\max }$ and sensitivity were higher at $10^{\circ} \mathrm{C}\left(10\right.$ and $20^{\circ} \mathrm{C}$ protocol, Figure 5C). These findings suggest that, if exposed to vasoconstrictors, these vessels would react more, increasing vasoconstriction and the afterload during ventricular ejection.

To understand these results, we also investigated the role of the endothelium on such responses. With the Krebs solution, as expected, the endothelial removal increased contractile responses to phenylephrine. Regarding CEL $\left(10\right.$ and $20^{\circ} \mathrm{C}$ protocol, Figure 6) and BHTK solutions (10 and $20^{\circ} \mathrm{C}$ protocol, Figure 7), endothelial removal increased both $R_{\max }$ and sensitivity at both temperatures but these changes were enhanced at 10 ${ }^{\circ} \mathrm{C}$ when compared to $20^{\circ} \mathrm{C}$. These findings suggest that, if for some other reason the endothelium is damaged, an increased vasoconstriction will occur at $10^{\circ} \mathrm{C}$. These results are highlighted in Figures 6D and 7D, that show dAUCs comparisons of concentration-response curves to phenylephrine under control conditions (Krebs at $37^{\circ} \mathrm{C}$ ) and after exposure to CEL and BHTK solutions.

However, when investigating the STH-1 solution (10 and 20 ${ }^{\circ} \mathrm{C}$ protocol, Figures $8 \mathrm{~A}$ and $\mathrm{B}$ ), we observed that the reactivity to phenylephrine was much higher at $20^{\circ} \mathrm{C}$ than at $10{ }^{\circ} \mathrm{C}$. In Figure 8D, dAUCs show that this behaviour is enhanced when comparing STH-1 solution exposure to control conditions $\mathrm{C}$ and to $10^{\circ} \mathrm{C}$. These findings suggest that, if the rings are exposed to 10 ${ }^{\circ} \mathrm{C}$ with a damaged endothelium, as the temperature increases, the reactivity to vasoconstrictors will increase dramatically, compromising left ventricular ejection by an increased afterload. 


\section{Celsior Solution}
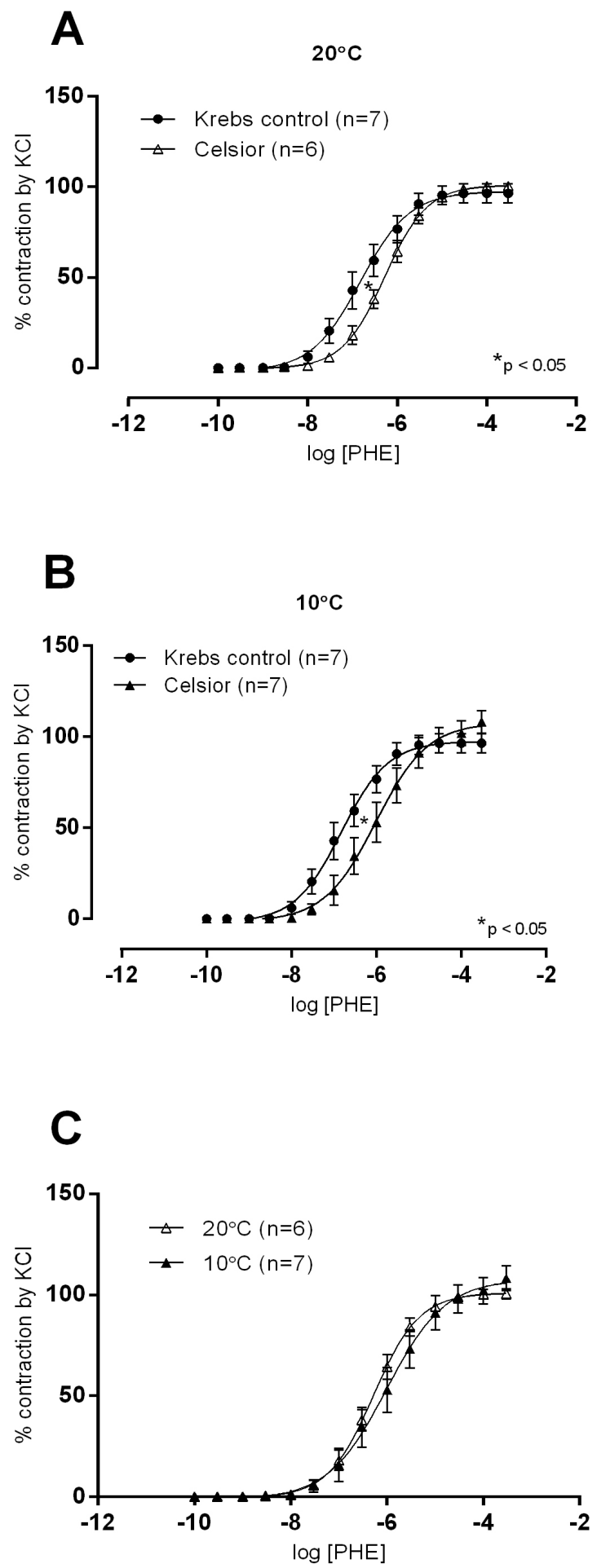

\section{Bretschneider-HTK}
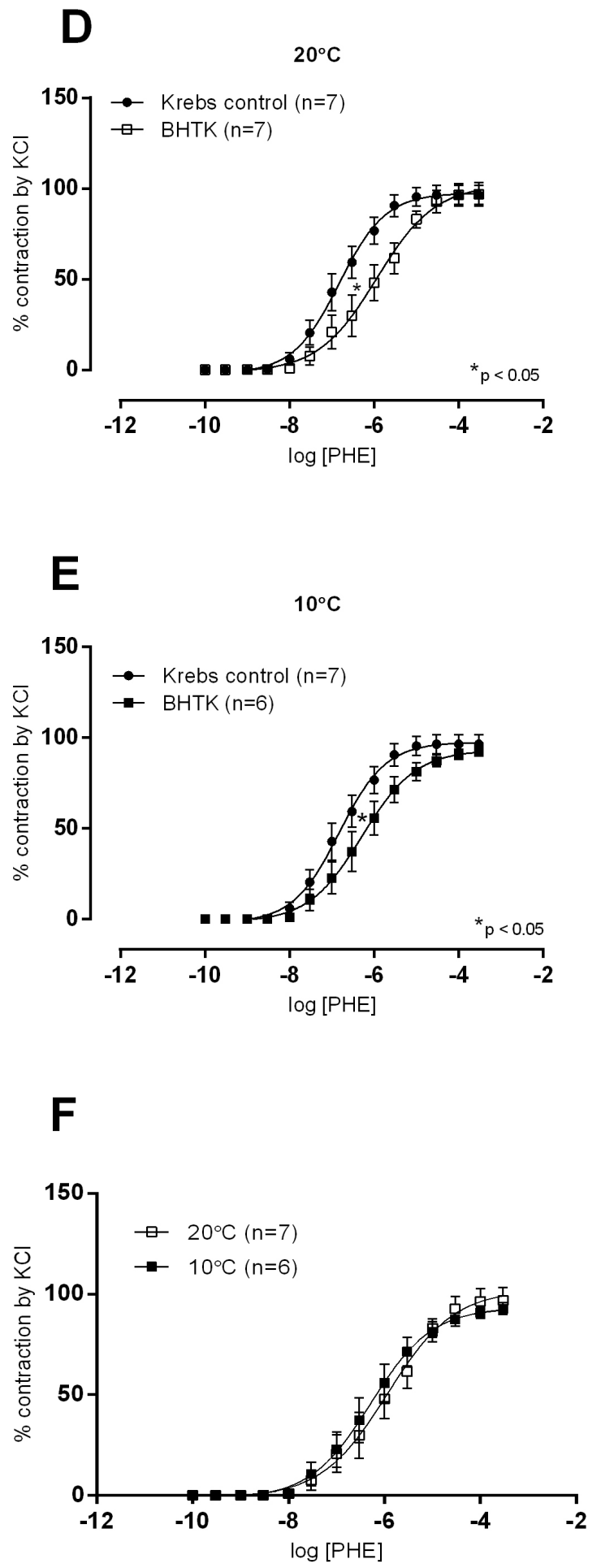

Fig. 4 - Concentration-response curves to phenylephrine (PHE) in isolated aortic rings of Wistar rats at $37^{\circ} \mathrm{C}$ in Krebs-Henseleit (Krebs control) solution after incubation for 1 hour in hypoxia at $20^{\circ} \mathrm{C}$ or $10^{\circ} \mathrm{C}$ with Celsior (A and B) or Bretschneider-HTK (BHTK) (D and E) solutions in pD2 and Rmax values. In C (Celsior) and F (BHTK), comparisons of concentration-response curves at $20^{\circ} \mathrm{C}$ or $10^{\circ} \mathrm{C}$. Results were expressed as mean \pm SEM. ${ }^{*} P<0.05$ vs. Krebs control solution, $t$-test for Rmax and pD2. The numbers in parentheses indicate the number of specimens used. 


\section{St Thomas}

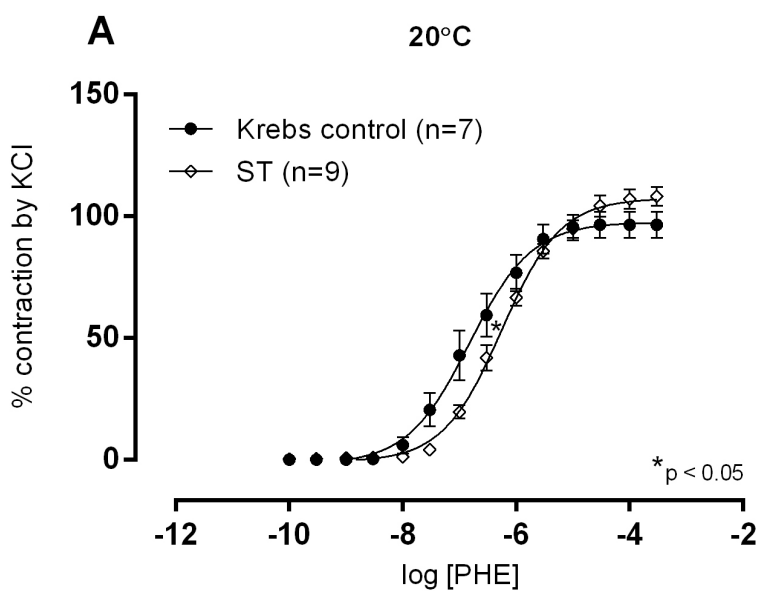

B

$10^{\circ} \mathrm{C}$

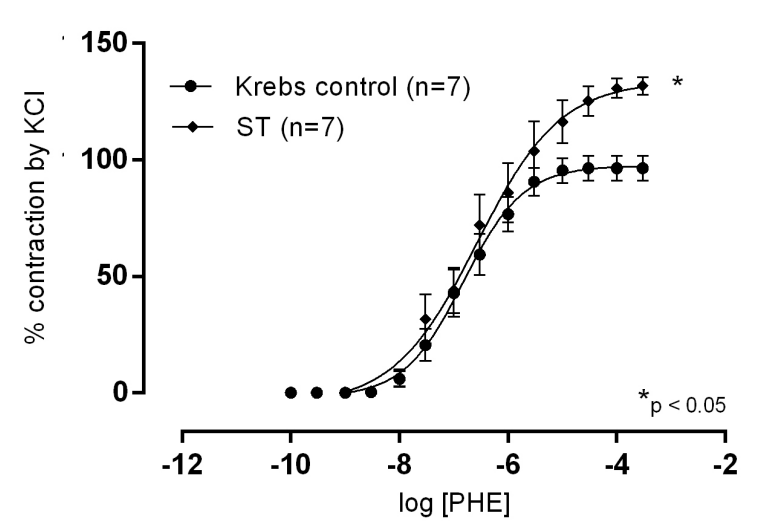

C

St Thomas $20^{\circ} \mathrm{C} \times 10^{\circ} \mathrm{C}$

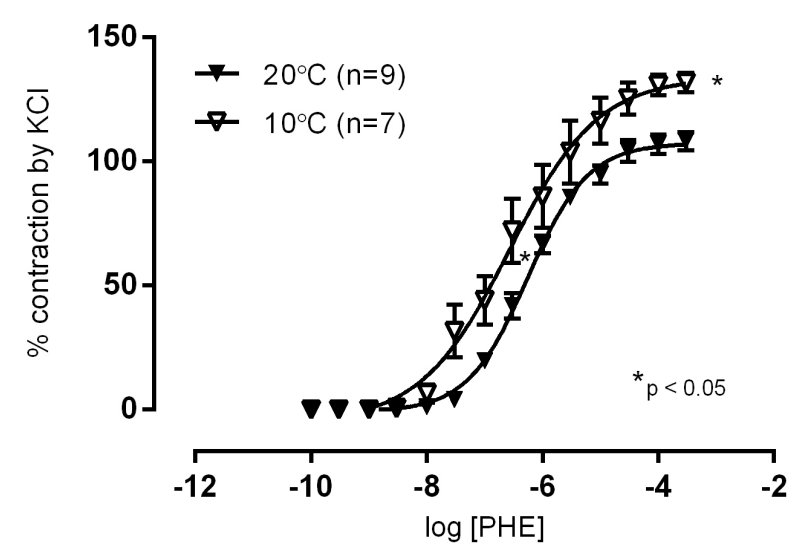

Fig. 5 - Concentration-response curves to phenylephrine (PHE) in isolated aortic rings of Wistar rats at $37{ }^{\circ} \mathrm{C}$ in Krebs-Henseleit (Krebs control) solution after incubation for 1 hour in hypoxia at $20^{\circ} \mathrm{C}$ (A) or 10 ${ }^{\circ} \mathrm{C}(B)$ of Rmax or pD2 of St. Thomas (ST) solution, compared to Krebs control solution, at $37^{\circ} \mathrm{C}$. In (C), comparison between concentrationresponse curves to PHE at $20^{\circ} \mathrm{C}$ or $10^{\circ} \mathrm{C}$. Results expressed as mean \pm SEM. ${ }^{*} P<0.05$ vs. CT (control); t-test for Rmax and $p D 2$. The numbers in parentheses indicate the number of specimens used.

\section{DISCUSSION}

Our results showed that the cardioprotective solutions used (Krebs, CEL, BHTK, and STH-1) administered at $37{ }^{\circ} \mathrm{C}$ for 1 hour of hypoxia did not damage the endothelium. However, Ringer, CEL and BHTK exposure reduced $\mathrm{KCl}$-induced contractions. The cardioprotective solutions used (CEL, BHTK and STH-1), administered at 10 and $20^{\circ} \mathrm{C}$ for 1 hour in hypoxia, also did not alter the endothelial function but the $\mathrm{KCl}$-induced contractions were similar.

When performing cardiac arrest for several purposes, myocardial ischemia takes place decreasing the production of adenosine triphosphate (ATP) and myocardial function. Prolonged ischemic periods may compromise myocardial viability even with methods of preservation. Thus, adequate myocardial preservation is a critical measure to ensure the viability of the heart in procedures performed with prolonged ischemic time ${ }^{[12]}$. Temperature reduction is frequently used for such purpose ${ }^{[13]}$ but so far there is no gold standard for myocardial protection ${ }^{[14]}$. Hypothermia is a standard strategy and, according to Cleveland et al. ${ }^{[15]}$, is the most important factor in myocardial protection.

Myocardial preservation studies have been the main goal to achieve good results and are mainly focused on the cardiac mechanical activity ${ }^{[5-7]}$. Moreover, studies regarding the effects of preservation solutions on vascular reactivity have been neglected. Therefore, if protective solutions damage the endothelium, they could be harmful to the heart, impairing blood flow and its mechanical recovery, increasing the vascular tone of conductance arteries and, consequently, the afterload against which the left ventricle has to eject.

We previously investigated the effects of cardioplegic or cardioprotective solutions, after 1 hour of hypoxia, at $37^{\circ} \mathrm{C}$, to select which solutions would be suitable to be evaluated at $10^{\circ} \mathrm{C}$ and $20^{\circ} \mathrm{C}$. KH, BHTK, STH-1, and CEL solutions were investigated covering a range of protective profiles such as different levels of potassium to keep the cell membrane depolarized and the myocardium arrested, free radicals scavengers, non-permeants to maintain osmotic pressure and to prevent edema, energy substrates, and buffers to prevent acidosis ${ }^{[16]}$. Our results showed that CEL and BHTK solutions, at such conditions (1 hour of hypoxia at $37^{\circ} \mathrm{C}$ ), reduced the vascular reactivity to phenylephrine.

Moreover, at $37{ }^{\circ} \mathrm{C}$, the concentration-response curves to phenylephrine showed a reduction of $R_{\max }$ after 1 hour of hypoxia under CEL and BHTK solutions, suggesting a reduction in vascular tone. This reduction was correlated with the reduction in the potassium concentration of the solutions, since CEL and BHTK solutions have a lower $\mathrm{KCl}$ concentration. Moreover, there was a positive correlation between $R_{\max }$ and the potassium concentration of the solutions, suggesting that the maintenance of a reduced vascular tone depended on the amount of potassium in the solutions used. In agreement, a recent study demonstrated that the CEL solution, which has a smaller $\mathrm{KCl}$ concentration, is the best for myocardial protection in isolated hearts submitted to cold static ischemia ${ }^{[17]}$.

Other studies on myocardial protection have shown benefits of the CEL and BHTK solutions ${ }^{[18]}$. Pereda et al. ${ }^{[19]}$ compared the 

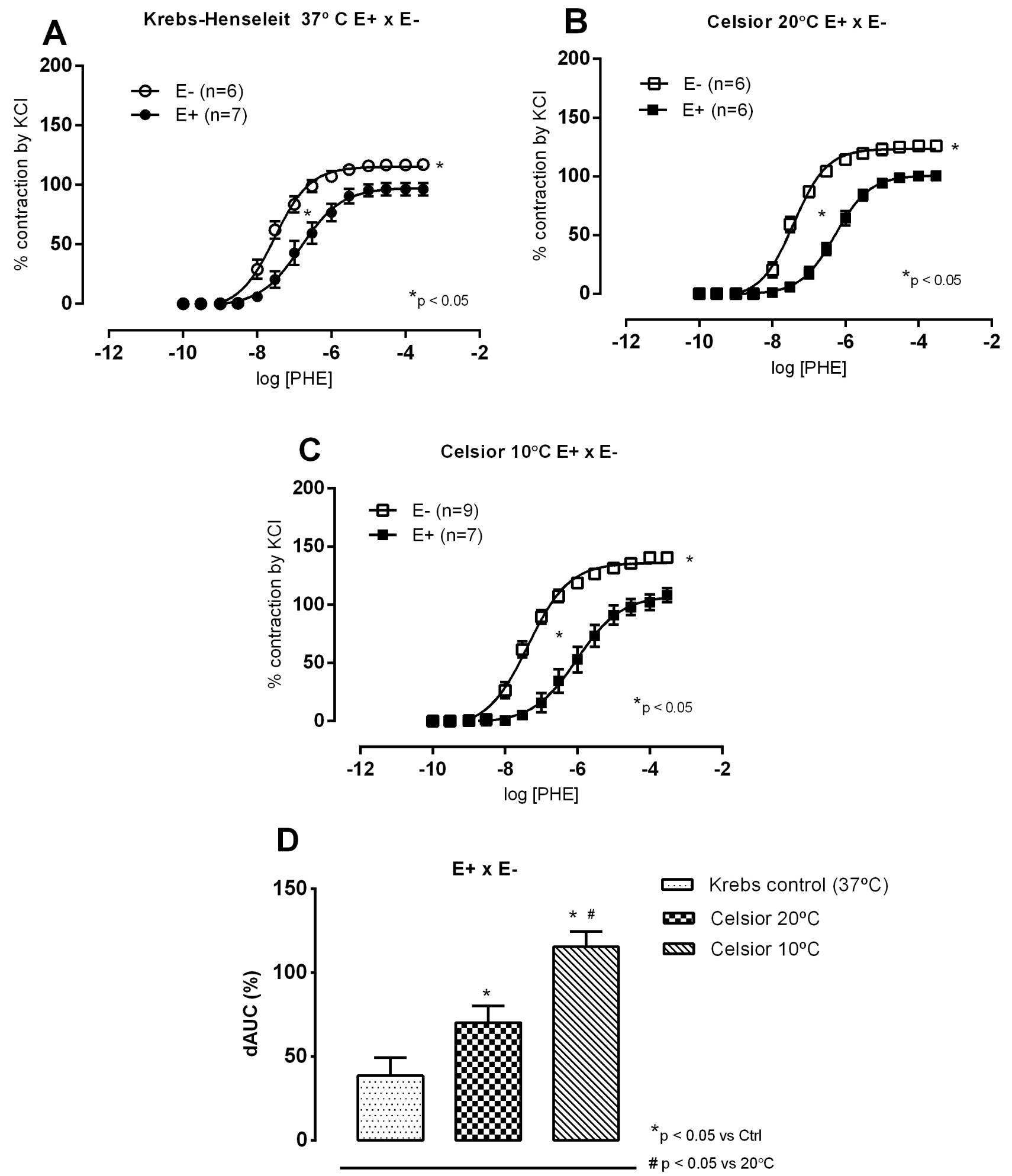

Fig. 6 - Concentration-response curves to phenylephrine (PHE) in the presence ( $E+$ ) and absence of endothelium (E-) in isolated aortic rings of Wistar rats at $37^{\circ} \mathrm{C}$ in Krebs-Henseleit solution, after incubation for 1 hour in hypoxia. In Krebs solution at $37^{\circ} \mathrm{C}(\mathrm{A})$ and at $20^{\circ} \mathrm{C}(\mathrm{B})$ or $10^{\circ} \mathrm{C}(\mathrm{C})$ with the Celsior solution; comparison of endothelial modulation by calculating the difference of area below the curve (dAUC), in \% (D). Results expressed as mean \pm SEM. In A, B and C, $P<0.05$ for comparisons of Rmax and $p D 2$ at $37^{\circ} \mathrm{C}, 20^{\circ} \mathrm{C}$ or $10^{\circ} \mathrm{C}, t$-test. In $D,{ }^{*} P<0.05$ for comparisons of Krebs control vs. CEL at $20^{\circ} \mathrm{C}$ or $10^{\circ} \mathrm{C}$, and ${ }^{\#} \mathrm{P}<0.05$ for comparisons of Krebs control vs. Celsior at $20^{\circ} \mathrm{C}$ or $10^{\circ} \mathrm{C}$, one-way ANOVA, Tukey test. The numbers in parentheses indicate the number of specimens used. 


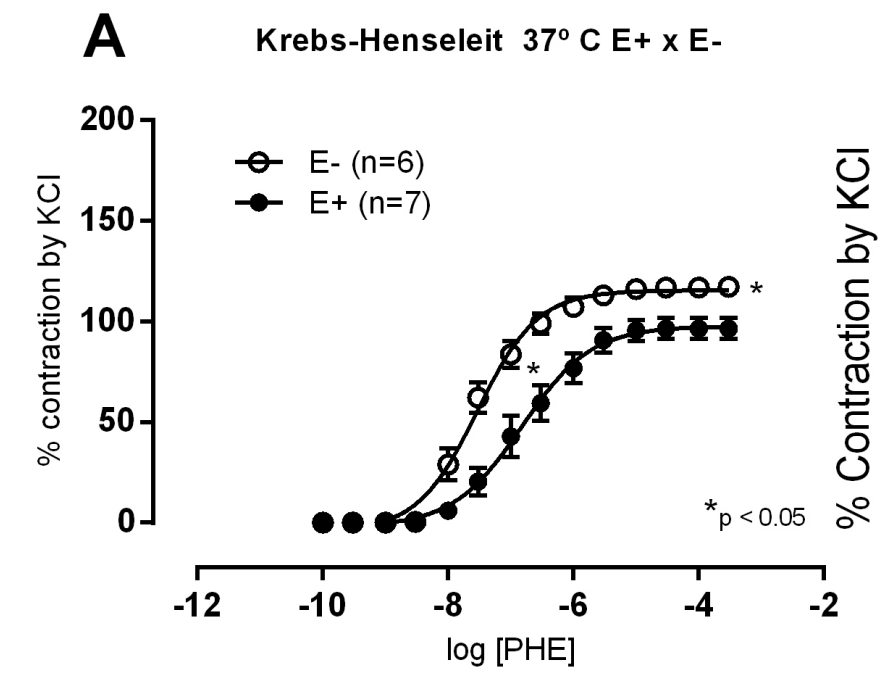

B Bretschneider-HTK $20^{\circ} \mathrm{CE}+\mathrm{xE}-$
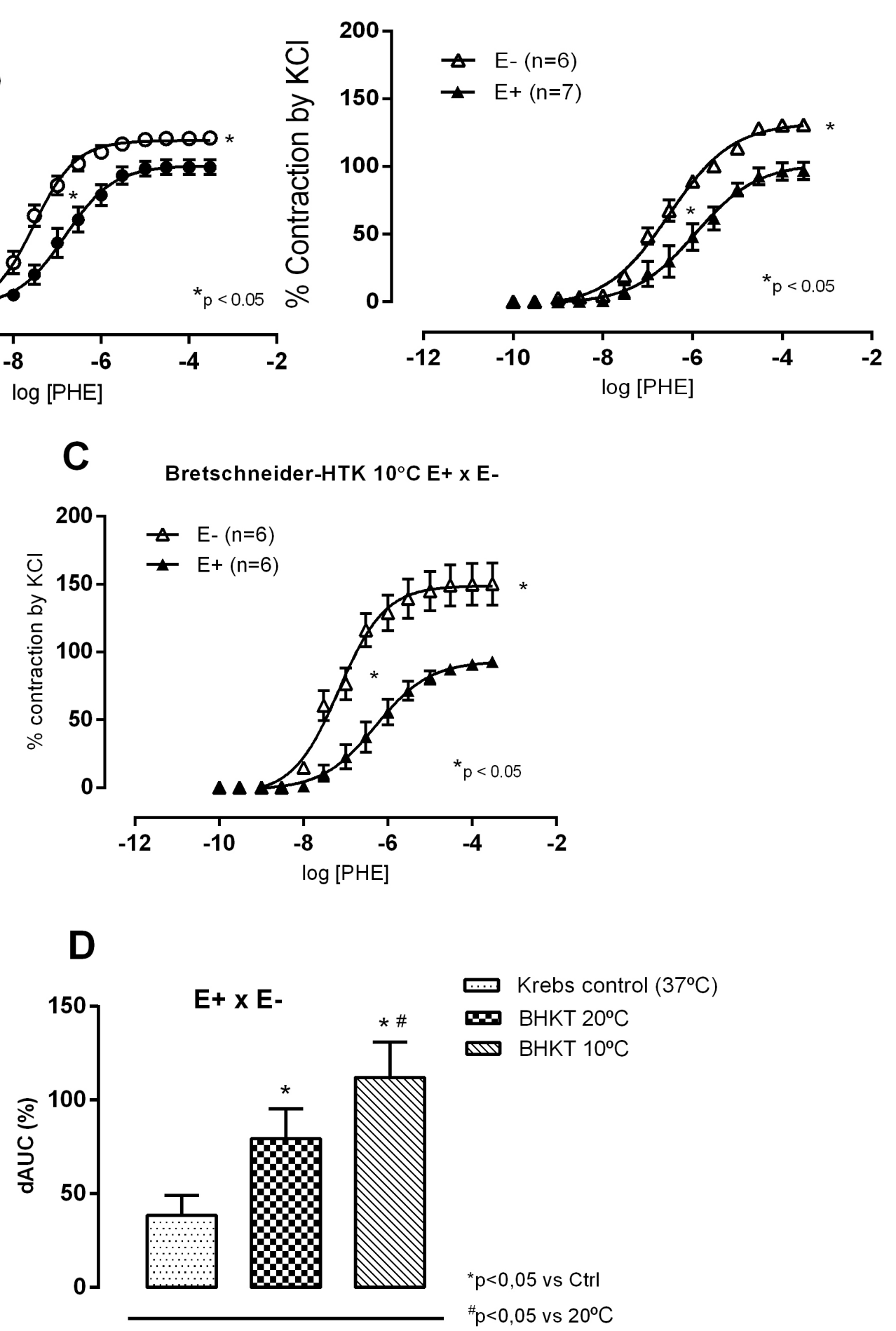

Fig. 7 - Concentration-response curves to phenylephrine (PHE) in the presence (E+) and absence of endothelium (E-) in isolated aortic rings of Wistar rats at $37^{\circ} \mathrm{C}$ in Krebs-Henseleit solution, after incubation for 1 hour in hypoxia. In Krebs solution at $37^{\circ} \mathrm{C}(\mathrm{A})$ and at $20^{\circ} \mathrm{C}(\mathrm{B})$ or $10^{\circ} \mathrm{C}$ (C) with the Bretschneider-HTK solution; comparison of endothelial modulation by calculating the difference of area below the curve (dAUC), in \% (D). Results expressed as mean \pm SEM. In A, B and C, $P<0.05$ for comparisons of Rmax and $p D 2$ at $20^{\circ} \mathrm{C}$ or $10^{\circ} \mathrm{C}, t$-test. In $D,{ }^{*} P<0.05$ for comparisons of CT vs. Bretschneider-HTK at $37^{\circ} \mathrm{C}, 20^{\circ} \mathrm{C}$ or $10^{\circ} \mathrm{C}$, and ${ }^{\#} \mathrm{P}<0.05$ for comparisons of Krebs control vs. BHTK at $20^{\circ} \mathrm{C}$ or $10^{\circ} \mathrm{C}$, oneway ANOVA, Tukey test. The numbers in parentheses indicate the number of specimens used. 

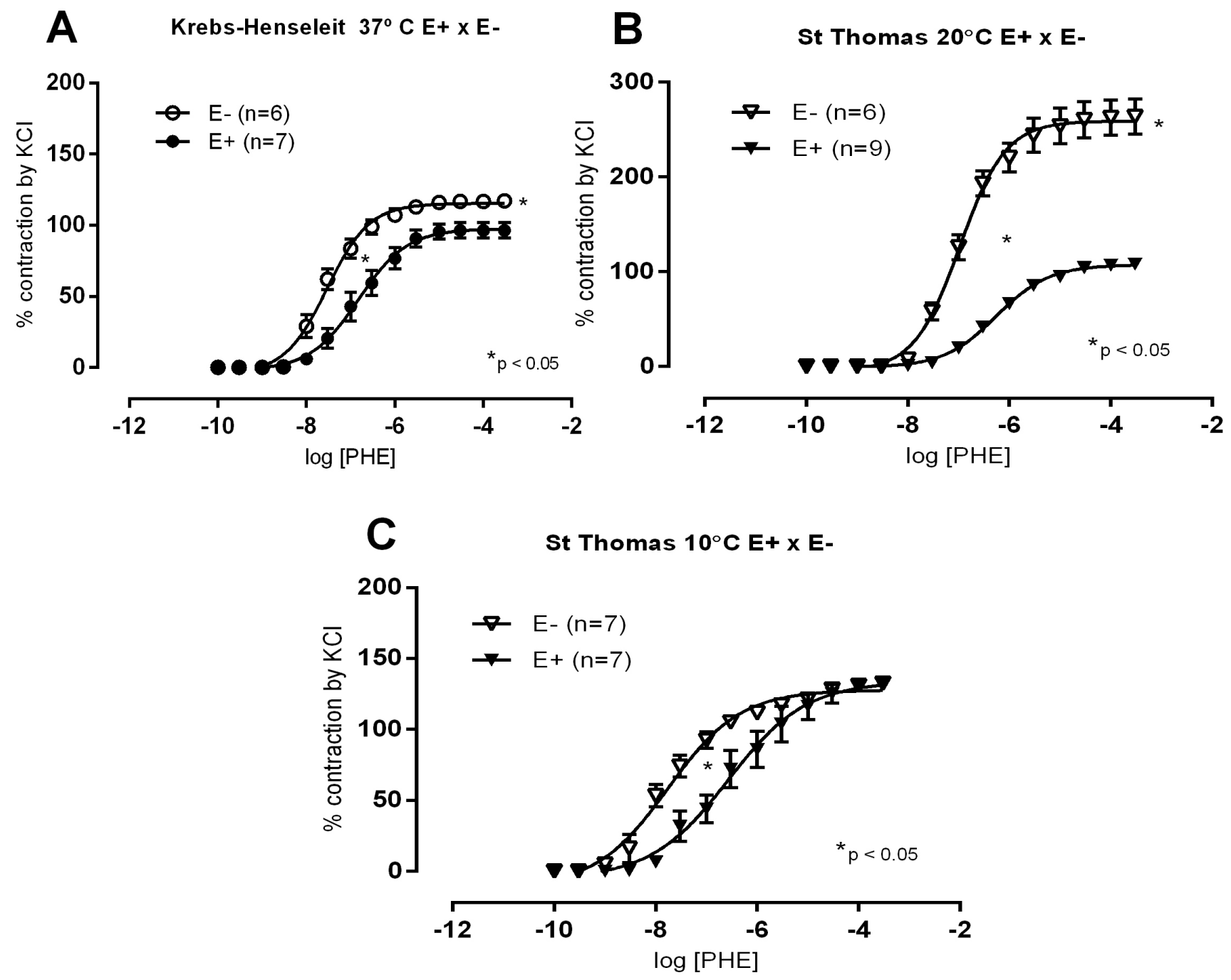

D

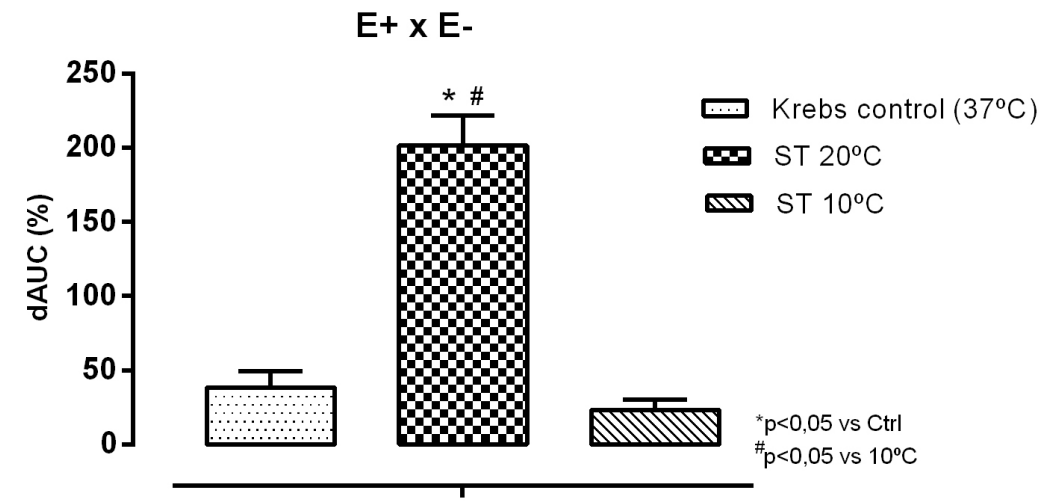

Fig. 8 - Concentration-response curves to phenylephrine (PHE) in the presence $(E+)$ and absence of endothelium (E-) in isolated aortic rings of Wistar rats at $37^{\circ} \mathrm{C}$ in Krebs-Henseleit solution, after incubation for 1 hour in hypoxia. In Krebs solution at $37^{\circ} \mathrm{C}(\mathrm{A})$ and at $20^{\circ} \mathrm{C}(\mathrm{B})$ or 10 ${ }^{\circ} \mathrm{C}(\mathrm{C})$ with St. Thomas solution; comparison of endothelial modulation by calculating the difference of area below the curve (dAUC), in \% (D). Results expressed as mean \pm SEM. In (A) and (B), $P<0.05$ for comparisons of Rmax and $p D 2$ at $37^{\circ} \mathrm{C}, 20^{\circ} \mathrm{C}$ or $10^{\circ} \mathrm{C}, t$-test. In (D), ${ }^{*} P<0.05$ for comparisons of Krebs control vs. St. Thomas at $20^{\circ} \mathrm{C}$ or $10^{\circ} \mathrm{C}$, and ${ }^{\#} \mathrm{P}<0.05$ for comparisons of CT vs. St. Thomas at $20^{\circ} \mathrm{C}$ or $10^{\circ} \mathrm{C}$, one-way ANOVA, Tukey test. The numbers in parentheses indicate the number of specimens used. 
performance of CEL with STH-1 solutions, demonstrating that they were not significantly different. Based on these findings, we investigated CEL, BHTK and STH-1 solutions after 1 hour of hypoxia at $10^{\circ} \mathrm{C}$ and $20^{\circ} \mathrm{C}$, and compared their effects with $\mathrm{KH}$ solution under control conditions, $37^{\circ} \mathrm{C}$ and no hypoxia. Concentrationresponse curves to phenylephrine, after exposure to 10 and $20^{\circ} \mathrm{C}$, showed a reduction of sensitivity but no changes in $R_{\max }$ after 1 hour of hypoxia under CEL and BHTK solutions, suggesting a reduction of sensitivity to an adrenergic-induced vasoconstrictor response. Regarding STH-1 solution, the sensitivity reduced after $20^{\circ} \mathrm{C}$ but not after exposure to $10{ }^{\circ} \mathrm{C}$ and $R_{\max }$ increased after exposure to $10{ }^{\circ} \mathrm{C}$ when compared to control conditions (Krebs at $37^{\circ} \mathrm{C}$ ). However, comparing concentration-response curves to phenylephrine, after exposure to $\mathrm{STH}-1$ solution at $10{ }^{\circ} \mathrm{C}$ and 20 ${ }^{\circ} \mathrm{C}$, an increase in sensitivity and $\mathrm{R}_{\max }$ was observed, suggesting an increased reactivity to adrenergic vasoconstrictors.

Endothelial damage (Figure 8) reinforced such findings. CEL and BHTK solutions increased $R_{\max }$ and sensitivity after endothelial removal at both temperatures but these changes were enhanced after exposure to $10^{\circ} \mathrm{C}$. However, after exposure to STH-1 solution, sensitivity and $R_{\max }$ were enhanced after exposure to $20^{\circ} \mathrm{C}$. Regarding coronary arteries, only a few reports presented effects of preservation solutions. Perrault et al.. ${ }^{[0]}$ investigated the effects of preservation solution on coronary relaxation, showing that only CEL better preserved endothelial dependent relaxation. Yang and $\mathrm{He}^{[11]}$ reviewed the effects on coronary arteries by preservation solutions, suggesting that they might have detrimental effects on the endothelium, mainly related to solutions containing high potassium concentrations. Then, if the rings exposed to $10^{\circ} \mathrm{C}$ have a damaged endothelium when temperature increases, the reactivity to vasoconstrictors will increase dramatically, which might impair blood flow and ventricular ejection.

Our findings suggest that $\mathrm{CEL}$ and BHTK solutions reduced vascular reactivity to phenylephrine in the aortic rings after exposure to $20^{\circ} \mathrm{C}$ and $10{ }^{\circ} \mathrm{C}$ with intact endothelium. When the endothelium was removed, an increase in $R_{\max }$ and sensitivity occurred after exposure to $10{ }^{\circ} \mathrm{C}$, suggesting that, at this low temperature, a dysfunctional endothelium will provide conditions for an increased vasoconstriction. Regarding the STH-1 solution, an increased reactivity to phenylephrine was observed, mainly after exposure to $10^{\circ} \mathrm{C}$. After endothelium removal, an enhanced reactivity was also observed after exposure to $20^{\circ} \mathrm{C}$. Once again, a dysfunctional endothelium will provide conditions for an increased vasoconstriction to occur.

We should emphasize that solutions that reduce vascular tone may have a better profile for myocardial perfusion and, consequently, cardiac recuperation. In addition, the reduction of conductance arteries tone reduces the afterload during ventricular ejection. In this context, this study showed that CEL and BHTK solutions reduced vascular tone, pointing towards an increase of blood flow and ventricular ejection, increasing cardioprotection.

\section{Limitations of the Study}

In this study, the endothelial factors involved in the changes described here were not investigated. This evaluation is under way. However, due to the original results presented here and the enormous amount of protocols that are necessary to study the role of several endothelial factors, this work was presented. The fact that patients undergoing surgical procedures usually have atherosclerosis and hypertension, conditions that might damage the endothelium, this will enhance the vascular smooth muscle contractility and, consequently, are enough to justify the presentation of this study.

\section{Conclusion}

In summary, our results show that hypoxia for 1 hour did not damage the endothelial function with the solutions used here but preservation under different temperatures might produce harmful consequences. However, CEL and BHTK solutions reduced vascular reactivity to phenylephrine at 37,20 and $10^{\circ} \mathrm{C}$. Moreover, this decreased vascular reactivity to phenylephrine correlated with the lower potassium concentration, which is lower in these solutions. It is important to emphasize that, although CEL and BHTK solutions were the ones that reduced the vascular tone in aortic rings, only CEL solution showed a better myocardial protection response in isolated rat hearts submitted to cold static ischemia ${ }^{[12]}$. In fact, solutions that reduce the vascular response to vasoconstrictors, a necessary condition to increase blood flow and reduce afterload, seem to have a better profile for better myocardial perfusion and protection and, consequently, for cardiac recovery. We must emphasize that cardiac surgeries are usually performed in patients with atherosclerotic disease or other conditions accompanied by endothelial dysfunction. In such conditions, the selection of myocardial protection procedures submitted to cold static ischemia needs special attention regarding adequate composition and temperature of the solution.

Financial support: This study was funded by the Brazilian Ministry of Science, Technology and Innovation, National Council for Scientific and Technological Development - Edital Universal/ CNPq No 44181/2014-9

Coordination for the Improvement of Higher Education Personnel - No 23038.006534/2016-93

Espírito Santo Research Foundation - PRONEX_FAPES/CNPq No 48511935/2009

\section{No conflict of interest.}




\section{Authors' roles \& responsibilities}

PRB Substantial contributions to the conception or design of the work; or the acquisition, analysis, or interpretation of data for the work; drafting the work or revising it critically for important intellectual content; agreement to be accountable for all aspects of the work in ensuring that questions related to the accuracy or integrity of any part of the work are appropriately investigated and resolved; final approval of the version to be published

DVV Substantial contributions to the conception or design of the work; or the acquisition, analysis, or interpretation of data for the work; drafting the work or revising it critically for important intellectual content; agreement to be accountable for all aspects of the work in ensuring that questions related to the accuracy or integrity of any part of the work are appropriately investigated and resolved; final approval of the version to be published

MRS Substantial contributions to the conception or design of the work; or the acquisition, analysis, or interpretation of data for the work; drafting the work or revising it critically for important intellectual content; agreement to be accountable for all aspects of the work in ensuring that questions related to the accuracy or integrity of any part of the work are appropriately investigated and resolved; final approval of the version to be published

MLL Substantial contributions to the conception or design of the work; or the acquisition, analysis, or interpretation of data for the work; drafting the work or revising it critically for important intellectual content; agreement to be accountable for all aspects of the work in ensuring that questions related to the accuracy or integrity of any part of the work are appropriately investigated and resolved; final approval of the version to be published

\section{REFERENCES}

1. Demmy TL, Biddle JS, Bennett LE, Walls JT, Schmaltz RA, Curtis JJ. Organ preservation solutions in heart transplantation--patterns of usage and related survival. Transplantation. 1997;63(2):262-9.

2. Jahania MS, Sanchez JA, Narayan P, Lasley RD, Mentzer RM Jr. Heart preservation for transplantation: principles and strategies. Ann Thorac Surg. 1999;68(5):1983-7. doi:10.1016/s0003-4975(99)01028-0.

3. Marshall VC. Renal preservation. In: Morris PJ. Kidney transplantation. Principles and practice. Philadelphia:WB Saunders; 200. p. 113-34.

4. Kumar MS, Samhan M, al Sabawi N, al Abdullah IH, Silva OS, White AG, et al. Preservation of cadaveric kidneys longer than 48 hours: comparison between euro-collins solution, UW solution, and machine perfusion. Transplant Proc. 1991;23(5):2392-3.

5. Koch A, Radovits T, Loganathan S, Sack FU, Karck M, Szabó GB. Myocardial protection with the use of L-arginine and $\mathrm{N}$-alphaacetyl-histidine. Transplant Proc. 2009;41(6):2592-4. doi:10.1016/j. transproceed.2009.06.150.
6. Maathuis $\mathrm{MH}$, Leuvenink HG, Ploeg RJ. Perspectives in organ preservation. Transplantation. 2007;83(10):1289-98. doi:10.1097/01. tp.0000265586.66475.cc.

7. Jacobs S, Rega F, Meyns B. Current preservation technology and future prospects of thoracic organs. Part 2: heart. Curr Opin Organ Transplant. 2010;15(2):156-9. doi:10.1097/MOT.0b013e328337343f.

8. Ning XH, Chen SH, Xu CS, Li L, Yao LY, Qian K, et al. Hypothermic protection of the ischemic heart via alterations in apoptotic pathways as assessed by gene array analysis. J Appl Physiol (1985). 2002;92(5):2200-7. doi:10.1152/japplphysiol.01035.2001.

9. Ning XH, Xu CS, Song YC, Childs KF, Xiao Y, Bolling SF, et al. Temperature threshold and modulation of energy metabolism in the cardioplegic arrested rabbit heart. Cryobiology. 1998;36(1):2-11. doi:10.1006/ cryo.1997.2054

10. Perrault LP, Nickner C, Desjardins N, Dumont E, Thai P, Carrier M. Improved preservation of coronary endothelial function with celsior compared with blood and crystalloid solutions in heart transplantation. J Heart Lung Transplant. 2001;20(5):549-58. doi:10.1016/s1053-2498(01)00242-x.

11. Yang Q, He GW. Effect of cardioplegic and organ preservation solutions and their components on coronary endothelium-derived relaxing factors. Ann Thorac Surg. 2005;80(2):757-67. doi:10.1016/j. athoracsur.2004.10.003.

12. Fiorelli Al, Stolf NA, Pego-Fernandes PM, Oliveira Junior JL, Santos RH, Contreras CA, et al. Recommendations for use of marginal donors in heart transplantation: Brazilian association of organs transplantation guideline. Transplant Proc. 2011;43(1):211-5. doi:10.1016/j. transproceed.2010.12.047.

13. Lima ML, Fiorelli Al, Vassallo DV, Batista PR, Simoes FV, Fiorim J, et al. Deleterious effect of hypothermia in myocardial protection against cold ischemia: a comparative study in isolated rat hearts. Transplant Proc. 2012;44(8):2326-32. doi:10.1016/j.transproceed.2012.07.007.

14. Hertz MI, Aurora P, Christie JD, Dobbels F, Edwards LB, Kirk R, et al. Scientific registry of the international society for heart and lung transplantation: introduction to the 2010 annual reports. J Heart Lung Transplant. 2010;29(10):1083-8. doi:10.1016/j.healun.2010.08.005.

15. Cleveland JC Jr, Meldrum DR, Rowland RT, Banerjee A, Harken AH. Optimal myocardial preservation: cooling, cardioplegia, and conditioning. Ann Thorac Surg. 1996;61 (2):760-8. doi:10.1016/0003-4975(95)01129-3.

16. Steenbergen C, Murphy E, Watts JA, London RE. Correlation between cytosolic free calcium, contracture, ATP, and irreversible ischemic injury in perfused rat heart. Circ Res. 1990;66(1):135-46. doi:10.1161/01. res.66.1.135.

17. Lima ML, Fiorelli Al, Gomes OM, Pinheiro BB, Da Silva MA, Porto LA, et al. Comparative analysis of the performance of various crystalloid cardioplegic solutions on myocardial protection after prolonged cold ischemia. Transplant Proc. 2011;43(1):80-3. doi:10.1016/j. transproceed.2010.12.044.

18. Bailey CP, Bolton HE, Jamison WL, Nichols HT. Commissurotomy for rheumatic aortic stenosis. I. Surgery. Circulation. 1954;9(1):22-31. doi:10.1161/01.cir.9.1.22.

19. Pereda D, Castella M, Pomar JL, Cartaña R, Josa M, Barriuso C, et al. Elective cardiac surgery using Celsior or St. Thomas No. 2 solution: a prospective, single-center, randomized pilot study. Eur J Cardiothorac Surg. 2007;32(3):501-6. doi:10.1016/j.ejcts.2007.05.021. 